\title{
Effects of expected vs. unexpected proximal US preexposure on taste-aversion learning
}

\author{
MICHAEL DOMJAN and GAIL A. GEMBERLING \\ University of Texas, Austin, Texas 78712
}

\begin{abstract}
Animals were first conditioned to expect lithium treatment following exposure to one taste solution (the $\mathrm{CS}+$ ) and to expect no drug treatment following exposure to another flavor (the CS-). All subjects then received a saccharin taste-aversion conditioning trial. In Experiment 1 , this conditioning trial was preceded $0,1,2,4$, or $6 \mathrm{~h}$ earlier by exposure to the CS+ flavor for independent groups. The CS+ exposure attenuated saccharin aversion learning if it occurred immediately before the saccharin conditioning trial but not if it occurred $1 \mathrm{~h}$ or more before conditioning. In Experiment 2, the saccharin conditioning trial was preceded 3 or $4.5 \mathrm{~h}$ earlier by a lithium injection. This proximal US preexposure injection was either unannounced (Li) or preceded by exposure to the $\mathrm{CS}+(\mathrm{CS}+\mathrm{Li})$ or the $\mathrm{CS}-(\mathrm{CS}-\mathrm{Li})$ stimuli. The US preexposure attenuated saccharin aversion learning in all cases. However, the interference effect was less when the preexposure injection was expected $(C S+L i)$ than when it was unexpected (CS-Li). This outcome could not be explained in terms of direct effects of the CS+ and CS- stimuli on the saccharin conditioning trial, and shows that the proximal US preexposure effect is a function of not only the drug dosage and preexposure interval, but also the anticipation of the drug pretreatment.
\end{abstract}

Rats readily learn to avoid novel-flavored solutions that are paired with aversive drug treatment (for reviews, see Barker, Best, \& Domjan, 1977; Milgram, Krames, \& Alloway, 1977). Nevertheless, there are various manipulations that can disrupt taste-aversion learning. One of these involves administering the drug unconditioned stimulus (US) once shortly before the taste-aversion conditioning trial (Cannon, Berman, Baker, \& Atkinson, 1975; Domjan \& Best, 1977). The attenuation of aversion conditioning that results from this treatment is referred to as the proximal US-preexposure effect.

Recent research has identified numerous circumstances in which the proximal US-preexposure effect occurs. Proximal preexposure disrupts aversion learning even if the preexposure US treatment is otherwise effective in producing a "backward" conditioned taste aversion (Domjan \& Best, 1977). The phenomenon occurs whether or not the conditioning drug treatment is administered before or after exposure to the CS flavor during the training trial (Domjan, 1978), and the interference effect is also evident when subjects are tested for their condi-

The research was supported by Grants MH 3078801 from the Public Health Service and BNS 77-01552 from the National Science Foundation. We are grateful to $M$. R. Best and J. Batson for their suggestions and encouragement. Requests for reprints should be addressed to Michael Domjan, Department of Psychology, University of Texas, Austin, Texas $\mathbf{7 8 7 1 2 .}$ tioned taste aversions while they are under the influence of the drug (Best \& Domjan, 1979). Finally, several experiments have shown that the disruption of conditioning is not influenced by the stimuli that accompany the preexposure drug treatment. For example, pairing the preexposure drug treatment with a novel flavor does not modify the interference with conditioning (Best \& Domjan, 1979), and the preexposure effect is not altered by either using different methods of drug administration for the preexposure and conditioning drug treatments (infusion of the drug without handling the animals or injection of the drug with a needle) or presenting the preexposure and conditioning drug treatments in different environments (the home cage vs. a darkened experimental chamber) (Domjan \& Best, Note 1).

To date, research on the proximal US-preexposure phenomenon has identified only two variables that influence the degree of interference with conditioning. The interference effect is directly related to the dose of the preexposure injection and is inversely related to the interval between US preexposure and the taste-aversion conditioning trial (Best \& Domjan, 1979; Cannon et al., 1975; Domjan, 1978; Domjan \& Best, 1977). The fact that the disruption of conditioning has been found to be influenced only by the preexposure interval and drug dosage suggests that the phenomenon is governed entirely by pharmacological variables. This conclusion is also encouraged by the fact that manipulations of the stimuli that 
accompany the preexposure drug treatment have not been found to influence the interference with conditioning (Best \& Domjan, 1979; Domjan \& Best, Note 1).

The present experiments were conducted as a part of our continuing search for nonpharmacological behavioral variables that might influence the proximal US-preexposure effect. One behavioral variable that has been found to control the effectiveness of drug treatments in a variety of other situations involves the extent to which the drug treatments are expected by the subject on the basis of stimuli that precede drug administrations. In a series of experiments, Siegel and his associates have shown that morphine is less effective when it is preceded by cues that reliably predict its administration than when the drug is administered when the subject does not expect it (Siegel, 1975, 1976, 1977, 1978; Siegel, Hinson, \& Krank, 1978). Similarly, lithium has been found to be less effective in conditioning flavor aversions when the drug is expected as compared with when the drug is not preceded by stimuli that reliably signal its occurrence (Gillan \& Domjan, 1977; Willner, 1978). The unconditioned suppressive effects of lithium on drinking are also reduced when the drug is expected (Domjan, Gillan, \& Gemberling, 1980).

The present investigation was conducted to determine whether expectancies can similarly influence the extent to which proximal drug preexposure interferes with taste-aversion conditioning. Lithium chloride was used as the drug US. Animals were first conditioned to expect lithium following exposure to one taste solution (the $\mathrm{CS}+$ ) and to expect no drug treatment following exposure to another flavor (the $\mathrm{CS}-$ ). After this initial differential conditioning, all subjects received a saccharin taste-aversion conditioning trial. For the proximal preexposure subjects, this conditioning trial was preceded several hours earlier by a lithium injection. In some cases, the preexposure drug treatment was signaled by exposure to the CS + flavor; in other cases, drug preexposure was given either without taste exposure or after exposure to the CS - flavor.

If expected drug preexposure is less effective than unexpected preexposure, then subjects whose preexposure injection is signaled by the CS + flavor should evidence less disruption of saccharin-aversion learning than subjects that receive unsignaled preexposure or a preexposure injection preceded by the CS - flavor. This prediction is a direct extension of results obtained in other experiments that have compared the effectiveness of expected and unexpected drug treatments. However, on the basis of the previous research, one cannot be fully assured that less disruption of conditioning will be evident with expected than with unexpected proximal US preexposure. Such an outcome will occur only if the $\mathrm{CS}+$ flavor that announces the preexposure drug injection does not also influence the effectiveness of the lithium injection that is administered later as a part of the saccharin conditioning trial. If exposure to the CS + flavor creates an expectancy for lithium that persists until the subsequent saccharin conditioning injection, then the effectiveness of the conditioning drug treatment may be attenuated, resulting in a reduction in saccharin-aversion learning (Gillan \& Domjan, 1977). Thus, evidence of less interference with conditioning produced by the expectancy of proximal US preexposure may be obscured by disruption of conditioning resulting from expectancy of the saccharin conditioning injection.

Whether or not the effects of drug expectancy on proximal US preexposure can be investigated independently of the direct effects of drug expectancy on saccharin conditioning depends on the relative time course of these two phenomena. If the US-preexposure effect and the direct effects of drug expectancy on conditioning have the same temporal characteristics, then an unconfounded examination of expected vs. unexpected proximal US preexposure is not possible. The temporal characteristics of the proximal US-preexposure effect have been documented in previous experiments (Best \& Domjan, 1979; Domjan, 1978; Domjan \& Best, 1977). However, the time course of the direct effects of drug expectancy on aversion conditioning is not yet known. Therefore, Experiment 1 was conducted to provide this information. The results showed that the direct effects of drug expectancy on saccharin conditioning are much shorter lasting than the effects of proximal US preexposure. This outcome made it possible in Experiment 2 to investigate the effects of expected vs. unexpected proximal US preexposure using procedures that minimized the direct effects of drug expectancy on conditioning.

\section{EXPERIMENT 1}

Gillan and Domjan (1977) have shown that saccharin aversion learning is disrupted if the saccharindrug conditioning trial is immediately preceded by exposure to a flavored solution (CS+) that was previously paired with the drug. Experiment 1 was conducted to determine the time course of this interference effect. The method was modeled after that used by Gillan and Domjan (1977). During the first phase of the experiment, all subjects received differential conditioning in which exposure to one taste solution (the $\mathrm{CS}+$ ) was followed by a lithium injection, and exposure to a second flavor (the $\mathrm{CS}-$ ) was given without drug treatment. All subjects then received a saccharin conditioning trial. For some subjects, this trial occurred without exposure to other 
tastes; for others, saccharin conditioning was preceded by exposure to the CS + flavor $0,1,2,4$, or $6 \mathrm{~h}$ earlier.

\section{Method}

Subjects and Preexperimental preparation. Sixty-three male Sprague-Dawley rats (Holtzman Co., Madison, Wisconsin, 200$250 \mathrm{~g}$ at the start of the experiment, were housed in individual wire-mesh cages with continuous access to Purina Rat Chow and water, except as noted below. Before the start of the experiment, the rats were anesthetized with ether and an oral fistula was implanted in a manner similar to that described by Domjan and Wilson (1972). A section of P.E. 205 polyethylene tubing was passed under the skin of the neck of the animals, with one end exiting at the back of the neck and the other end exiting just anterior to the right molar teeth. The two ends were flared, and the end in the oral cavity was held in place by a polyethylene washer. In addition, the tubing was secured by a wire suture attached to the oral end and affixed to subcutaneous tissue in the cheek.

After the operation, the drinking water of the animals was mixed with Terramycin for the remainder of the experiment to help control infections. Each subject also received a .2-ml intramuscular injection of Combiotic on each of the first 5 days after the operation. Upon recovery, access to water was limited to 30 min daily. On days of experimental treatments, this water access followed the experimental manipulations by at least $1.5 \mathrm{~h}$.

Adaptation. After 5 days on the water-deprivation schedule, four sessions of adaptation to the oral infusion procedure were conducted on successive days. During these sessions, each rat had its cannula attached to a syringe in a Harvard Model 941 infusion pump via flexible Tygon B-44-3 tubing, was placed in a specially constructed cage surrounded by high walls, and received tap water infused through the fistula into the oral cavity for $5 \mathrm{~min}$ at a rate of $1.2 \mathrm{ml} / \mathrm{min}$. All oral infusions throughout the experiment were conducted with these parameters, which allowed the subjects to ingest the infused fluids comfortably (Domjan \& Wilson, 1972).

Differential conditioning. Beginning several days after adaptation to the infusion procedure, six $\mathrm{CS}+$ and $\mathrm{CS}-$ trials were conducted in a strictly alternating sequence with an intertrial interval of 1-2 days, starting with the CS + . On CS + trials, subjects received a 5 -min oral infusion of the $\mathrm{CS}+$ flavor, followed within $3 \mathrm{~min}$ by a $1.8-\mathrm{meq} / \mathrm{kg}$ intraperitoneal injection of $.15 \mathrm{M}$ lithium chloride. On CS- trials, subjects received a 5 -min oral infusion of the CS- flavor and no injections were administered after the taste presentation. A $1 \%(\mathrm{wt} / \mathrm{vol})$ solution of sodium chloride in tap water and a $3 \%$ ( $\mathrm{vol} / \mathrm{vol})$ solution of cider vinegar (4.5\% acidity) served as the CS + and CS - flavors. For 31 subjects, the CS + was the vinegar flavor and the CS - was the sodium flavor; for the other 32 subjects, these flavor assignments were reversed. Immediately before each taste infusion, the fistula and oral cavity were rinsed with $3-5 \mathrm{ml}$ of tap water, and the animals were thoroughly dried with a paper towel after each taste infusion to remove any infused fluids which they did not swallow and which may have gotten on their fur.

Saccharin conditioning. Each subject was assigned to one of six groups for saccharin conditioning. Group TAC (taste aversion control: $\mathrm{n}=11.6$ with vinegar as the $\mathrm{CS}+$ ) received a 5 -min infusion exposure to a $.5 \%$ (wt/vol) solution of sodium saccharin, followed within $3 \mathrm{~min}$ by a $1.8 \mathrm{meq} / \mathrm{kg}$ injection of .15 M lithium chloride. Each of the other groups received the same saccharinlithium pairing. For Group $\mathrm{O}(\mathrm{n}=11,5$ with vinegar as the $\mathrm{CS}+$ ), the saccharin-lithium pairing was immediately preceded by a 5 -min infusion exposure to the $\mathrm{CS}+$ flavor. In contrast, Group 1 ( $\mathrm{n}=11,5$ with vinegar as the $\mathrm{CS}+)$, Group $2(\mathrm{n}=10,5$ with vinegar as the $C S+)$, Group $4(n=10,5$ with vinegar as the $C S+)$, and Group $6(\mathrm{n}=10,5$ with vinegar as the $\mathrm{CS}+)$ received a 5 -min exposure to the CS+ flavor $1,2,4$, and $6 \mathrm{~h}$ before the saccharinlithium pairing, respectively. Every effort was made to insure that peripheral traces of the CS + flavor did not exist between exposure to the $\mathrm{CS}+$ and the saccharin conditioning trial. The subjects were thoroughly wiped dry after the CS + infusion, and, for Groups 1 , 2,4 , and 6, the CS+ infusion stands were removed from the experimental room during the saccharin conditioning trial. Subjects did not receive access to water in the home cage on the saccharin conditioning day.

Postconditioning test. Seven to 9 days after saccharin conditioning, each subject received a 120 -min one-bottle test with the saccharin solution in the home cage after $23.5 \mathrm{~h}$ water deprivation. The test fluid was presented in inverted graduated-centifuge tubes fitted with rubber stoppers and stainless steel drinking spouts.

\section{Results}

The amount of saccharin each group drank during the postconditioning test is presented in Figure 1. A monotonic gradient in saccharin consumption occurred as a function of the interval between exposure to the previously conditioned CS + flavor and saccharin during the saccharin conditioning trial. Group O, which had been exposed to the CS + immediately before saccharin conditioning, displayed the weakest aversion to the saccharin flavor. Progressively stronger aversions were evident in Groups 1,2 , 4 , and 6 , which had received progressively longer intervals between exposure to the $\mathrm{CS}+$ flavor and the saccharin conditioning trial.

The saccharin test intakes were first evaluated with a 6 by 2 analysis of variance in which the first factor was the six experimental groups and the second factor was the assignment of vinegar and sodium chloride as the $\mathrm{CS}+$ flavor during the differential

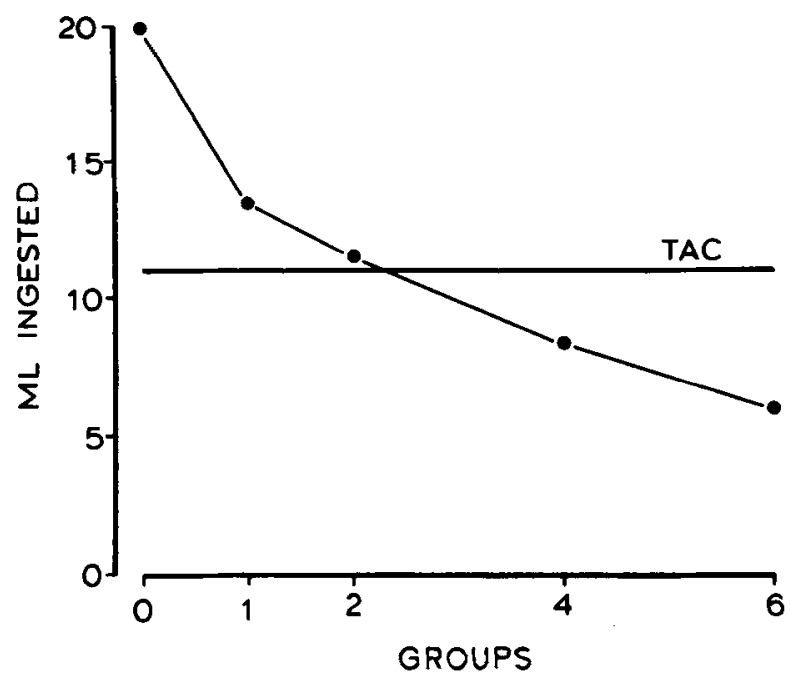

Figure 1. Amount of saccharin ingested during the postconditioning test in Experiment 1. The saccharin conditioning trial was conducted $0,1,2,4$, and $6 \mathrm{~h}$ after independent groups were exposed to a flavored solution (CS + ) that had been paired previously with lithium treatment. Group TAC (taste-aversion control) did not receive exposure to the $\mathrm{CS}+$ flavor on the saccharin conditioning day. 
conditioning phase of the experiment. This analysis confirmed that significant group differences existed in saccharin aversion learning $[F(5,51)=4.78, p<.01]$. However, neither the main effect of the CS + flavor assignments nor the interaction between groups and $\mathrm{CS}+$ flavors was significant $[\mathrm{F}(1,51)=2.46$, and $F(5,51)=2.08$, respectively, ps $>.05]$. Subsequent comparisons with the Newman-Keuls test $(p<.05)$ indicated that Group $\mathrm{O}$ drank significantly more saccharin during the test session than any of the other groups, which did not differ among themselves.

\section{Discussion}

The observation that Group $\mathrm{O}$ acquired significantly weaker saccharin aversions than Group TAC confirms that exposure to a previously conditioned stimulus (the $\mathrm{CS}+$ ) attenuates taste-aversion learning (Gillan \& Domjan, 1977). This interference effect no doubt resulted from the drug-conditioned properties of the CS + flavor, because previous research has shown that the effect is diminished by extinction of the $\mathrm{CS}+$ before saccharin conditioning and does not occur if the saccharin conditioning trial is conducted after exposure to the CS - instead of the CS + flavor (Gillan \& Domjan, 1977).

Although exposure to the CS + flavor immediately before the saccharin conditioning trial significantly attenuated aversion learning, disruption of conditioning was not observed if exposure to the CS + preceded saccharin conditioning by $1 \mathrm{~h}$ or more. This rapid decay of the interference effect may reflect the fact that during the initial differential conditioning phase, the CS + flavor was followed within $3 \mathrm{~min}$ by lithium treatment. Therefore, the subjects may have learned to expect lithium malaise very shortly after exposure to the CS + . Perhaps disruption of conditioning would occur with longer intervals between exposure to the $\mathrm{CS}+$ and saccharin conditioning if the initial conditioning of the $\mathrm{CS}+$ flavor were conducted with a longer interval between contact with the CS + flavor and drug administration. An alternative interpretation of the temporal gradient of interference observed in the present experiment is that it reflects the decay of the trace of the CS+ flavor. If this is true, the interference effect may not be prolonged by the use of longer intervals between $\mathrm{CS}+$ and drug treatment during the initial phase of the experiment.

The temporal gradient of interference observed in the present experiment is much shorter than the temporal gradient observed in previous studies of the effects of proximal US preexposure. While no disruption of conditioning was observed when exposure to the CS + preceded the saccharin conditioning trial by $1 \mathrm{~h}$ or more, previous experiments have shown that drug treatment disrupts conditioning at preexposure intervals as long as 3-6 h (Best \& Domjan, 1979). Therefore, by using a sufficiently long preexposure interval, it should be possible to investigate the effects of expected US preexposure on saccharin conditioning while minimizing any direct effects of the $\mathrm{CS}+$ that announces the preexposure drug injection.

\section{EXPERIMENT 2}

Experiment 1 confirmed that exposure to a previously conditioned $\mathrm{CS}+$ can reduce the effectiveness of a drug US in conditioning a saccharin aversion (cf. Gillan \& Domjan, 1977). Experiment 2 was designed to determine whether expectations can also influence the extent to which proximal drug preexposure interferes with taste-aversion conditioning. Independent groups were given a lithium injection 3 and $4.5 \mathrm{~h}$ before the saccharin conditioning trial. For some subjects, this proximal preexposure treatment was announced by exposure to a previously conditioned $\mathrm{CS}+$ flavor, whereas for others, it was paired with either exposure to a CS - flavor or no stimulation. Because the CS + and CS - flavors were presented 3 and $4.5 \mathrm{~h}$ before the saccharin conditioning trial, these stimuli were not expected to influence saccharin aversion learning directly (cf. Experiment 1).

As in Experiment 1, the first phase of Experiment 2 involved discriminative conditioning in which the $\mathrm{CS}+$ flavor was repeatedly paired with lithium and the CS - flavor was presented in the absence of drug treatment. Therefore, none of the subjects were naive with respect to the drug when the saccharin conditioning and the proximal US-prexposure treatments were conducted. This contrasts with previous published experiments on the proximal US-preexposure effect, all of which were done with drug-naive animals. However, it seemed unlikely that the drug exposure the subjects received during the initial discriminative conditioning phase would complicate interpretation of the proximal US-preexposure effects observed in the present experiment. First, every subject received the identical drug treatments in the initial phase of the experiment. Therefore, group differences could not be attributed to this factor. Second, each drug injection in the initial phase was paired with exposure to a novel flavored solution (the $\mathrm{CS}+$ ). Previous experiments have shown that exposure to a drug under such circumstances does not influence subsequent taste-aversion conditioning (Mikulka, Leard, \& Klein, 1977; Revusky, Parker, Coombes, \& Coombes, 1976). Finally, no significant differences in the proximal US-preexposure effect have been found in a systematic comparison of drugnaive and drug-experienced subjects (Best \& Domjan, Note 2).

\section{Method}

Twelve female and 53 male Sprague-Dawley rats were used. The method was identical to that of Experiment 1, except as specified below. After recovery from the fistula operation and adaptation to the daily 23.5-h water deprivation schedule, the subjects received four sessions of adaptation to the oral infusion procedure. Six 
$\mathrm{CS}+$ and six CS - differential conditioning trials were then conducted in a strictly alternating sequence. For 32 subjects, the $3 \%$ vinegar solution served as the CS + flavor and the $1 \%$ sodium chloride solution served as the CS - stimulus. For the other 33 subjects, these flavor assignments were reversed.

Three days after the differential conditioning phase, each subject received a saccharin conditioning trial which consisted of a 5 -min infusion exposure to a $.5 \%$-saccharin solution, followed within $3 \mathrm{~min}$ by an intraperitoneal injection of $1.8 \mathrm{meq} / \mathrm{kg}, .15 \mathrm{M}$ lithium chloride. For Group TAC (taste-aversion control: 1 female, 8 males, 4 subjects with vinegar as the CS +), the saccharin-lithium pairing was the only treatment on the conditioning day. In contrast, each of the other groups received signaled or unsignaled lithium treatment 3 or $4.5 \mathrm{~h}$ before the saccharin conditioning trial. Group $3 \mathrm{Li}$ ( 1 female, 8 males, 4 subjects with vinegar as the $\mathrm{CS}+$ ) received a $1.8-\mathrm{meq} / \mathrm{kg}$ lithium injection $3 \mathrm{~h}$ before being injected with lithium following the saccharin exposure. Group 3CS + Li ( 2 females, 8 males, 5 subjects with vinegar as the $\mathrm{CS}+$ ) also received a $1.8-\mathrm{meq} / \mathrm{kg}$ lithium injection $3 \mathrm{~h}$ before its saccharin conditioning drug injection, but, for these animals, the lithium preexposure was administered immediately after a 5 -min infusion exposure to the CS+ flavor. Group $3 \mathrm{CS}-\mathrm{Li}(2$ females, 7 males, 4 subjects with vinegar as the CS + ) was treated the same way as Group $3 \mathrm{CS}+\mathrm{Li}$, except that the lithium preexposure for this group occurred immediately after a 5-min infusion exposure to the CS- flavor. The remaining three groups of animals were treated the same way as Groups $3 \mathrm{Li}, 3 \mathrm{CS}+\mathrm{Li}$, and $3 \mathrm{CS}-\mathrm{Li}$, except that, for these animals, the lithium preexposure injection was administered $4.5 \mathrm{~h}$ before the saccharin conditioning drug injection: Group $4.5 \mathrm{Li}(2$ females, 7 males, 5 subjects with vinegar as the $\mathrm{CS}+$ ), Group $4.5 \mathrm{CS}+\mathrm{Li}$ ( 2 females, 7 males, 5 subjects with vinegar as the CS+), and Group 4.5CS-Li (2 females, 8 males, 5 subjects with vinegar as the CS + ). As in Experiment 1 , the subjects were thoroughly dried after the $\mathrm{CS}+$ and CS - taste infusions, and every effort was made to insure that peripheral traces of the CS + and CS - flavors were not present during the saccharin conditioning trial.

Three to 4 days after the saccharin conditioning trial, each subject received a 120 -min one-bottle test with the saccharin solution in the home cage after $23.5 \mathrm{~h}$ water deprivation. Starting 4-7 days after the saccharin test, each subject received two $30-\mathrm{min}$ one-bottle tests, separated by 2 days in the home cage. The CS + solution was available during one of these test sessions and the CS solution was available suring the other session. The order of these two tests was counterbalanced as closely as possible.

\section{Results}

Differential conditioning. The differential conditioning phase of the experiment established a strong differential response to the CS + and CS - flavors. Each subject drank substantially more of the CS than of the CS + flavor during the final test sessions of the experiment. The overall mean intake of the CS - flavor during these sessions was $21.6 \mathrm{ml}$, and the overall mean intake of the CS + flavor was $.5 \mathrm{ml}$.

Saccharin aversion conditioning. The amount of fluid each group drank during the postconditioning saccharin test is presented in Figure 2. Group TAC, which did not receive lithium treatment before the saccharin conditioning trial, drank the least amount of saccharin during the test session. Preconditioning exposure to lithium attenuated saccharin aversion learning at both the 3.0 - and 4.5 -h preexposure intervals. Furthermore, this interference with conditioning was influenced by the circumstances of lithium preexposure. Less disruption of conditioning

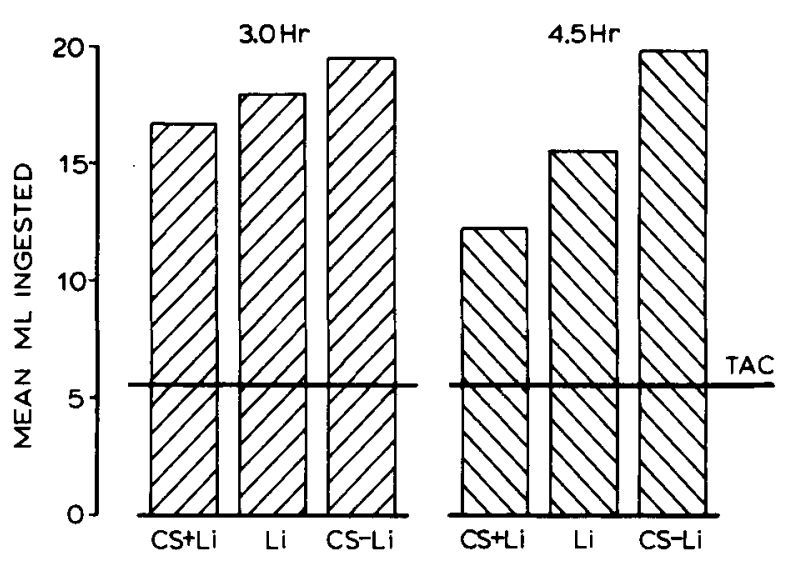

Figure 2. Amount of saccharin ingested during the postconditioning test in Experiment 2. The saccharin conditioning trial was preceded 3 or 4.5 h earlier by a lithium injection which was either unannounced (Li) or was preceded by exposure to a previously conditioned $\mathrm{CS}+(\mathrm{CS}+\mathrm{Li})$ or $\mathrm{CS}-(\mathrm{CS}-\mathrm{Li})$ flavor. Group TAC (taste-aversion control) did not receive exposure to lithium before the saccharin conditioning trial.

occurred when the preexposure drug treatment was paired with exposure to the CS + flavor than when the lithium preexposure was paired with the CSflavor. Preexposure without contact with either the $\mathrm{CS}+$ or $\mathrm{CS}-$ tastes produced intermediate effects.

The saccharin test intakes of the six drug-preexposed groups were first evaluated with a 2 by 3 analysis of variance. This analysis indicated that there was a significant effect of the three preexposure treatments, $\mathrm{CS}+\mathrm{Li}, \mathrm{Li}$, and $\mathrm{CS}-\mathrm{Li}[\mathrm{F}(2,50)=5.18, \mathrm{p}<.01]$. However, the effect of the two preexposure test intervals $(3.0$ and $4.5 \mathrm{~h})$ was not significant $[\mathrm{F}(1,50)=2.81$, $\mathrm{p}>.05]$. The interaction between the preexposure treatments and the preexposure interval was also not significant $[F(2,50)=1.12, p>.05]$. This last finding indicates that the three preexposure treatments did not have significantly different effects at the two preexposure intervals. Therefore, data for the two preexposure intervals were combined for each preexposure treatment in later analyses.

To further evaluate the effects of the circumstances of drug preexposure on taste-aversion conditioning, the three preexposure treatments $(\mathrm{CS}+\mathrm{Li}, \mathrm{Li}$, and $\mathrm{CS}-\mathrm{Li}$ ) were included with the nonpreexposed Group TAC in a one-way analysis of variance. This analysis confirmed that different levels of aversion resulted from the various saccharin conditioning procedures $[F(3,61)=17.96, p<.01]$. Subsequent comparisons with the Newman-Keuls test $(p<.05)$ indicated that each lithium-preexposed group learned a significantly weaker saccharin aversion than the nonpreexposed Group TAC. However, subjects that received preexposure treatment $\mathrm{CS}+\mathrm{Li}$ learned significantly stronger saccharin aversions than subjects that received preexposure treatment $\mathrm{CS}-\mathrm{Li}$. Lithiumalone preexposure did not result in effects signifi- 
cantly different from either $\mathrm{CS}+\mathrm{Li}$ or $\mathrm{CS}-\mathrm{Li}$ preexposure.

\section{Discussion}

The results of the present experiment confirm that US preexposure several hours before conditioning disrupts taste-aversion learning (Best \& Domjan, 1979; Domjan, 1978; Domjan \& Best, 1977). The interference effects were rather large, despite the fact that the subjects had extensive experience with lithium prior to the saccharin conditioning day because of the initial phase of differential taste-aversion conditioning. For example, Groups $3 \mathrm{Li}$ and $4.5 \mathrm{Li}$ drank approximately three times as much saccharin during the postconditioning test as the nonpreexposed Group TAC, which did not receive proximal US preexposure. This outcome confirms that the proximal US preexposure effect is evident in drug-experienced subjects (Best \& Domjan, Note 2 ).

Previous research was successful in identifying only two variables that influenced the proximal US-preexposure effect: the dose of the preexposure injection and the interval between preexposure and conditioning (Best \& Domjan, 1979; Cannon et al., 1975; Domjan, 1978; Domjan \& Best, 1977). This research also showed that certain environmental manipulations, such as pairing the preexposure injec tion with a novel flavor or altering the drug administration cues between preexposure and conditioning, do not influence the interference effect (Best \& Domjan, 1979; Domjan \& Best, Note 1). On the basis of these results, one might conclude that the proximal US-preexposure effect is mediated by physiological processes that are immune to the influence of behavioral variables. The results of the present experiments indicate that such a conclusion is unwarranted. Preexposure drug treatments announced by a signal for the drug (a CS + ) were less effective in disrupting conditioning than drug treatments announced by a stimulus that had been previously presented without drug injections (CS - ). Thus, in addition to temporal and dosage variables, the proximal USpreexposure effect is also influenced by expectation of the preexposure drug treatment.

The stronger aversion learning observed in subjects that received US preexposure preceded by contact with the $\mathrm{CS}+$, as compared to the CS -, flavor cannot be attributed to a direct effect of these stimuli on the saccharin conditioning trial. First, the results of Experiment 1 suggest that the direct effects of contact with the CS + require that the saccharin conditioning trial be conducted within $1 \mathrm{~h}$ of the CS+ exposure. Therefore, such effects are not expected with the long (3-4.5 h) preexposure intervals used in the present experiment. Second, if the CS + and CS stimuli directly influenced the effectiveness of the saccharin conditioning trial, weaker aversions should have been learned after $\mathrm{CS}+\mathrm{Li}$ than after $\mathrm{CS}-\mathrm{Li}$ preexposure (Gillan \& Domjan, 1977). The fact that the opposite outcome was observed strongly suggests that the present results were mediated by the influence of the CS+ and CS - flavors on the preexposure drug treatment.

The finding that proximal US preexposure is less effective in disrupting conditioning when it is expected $(\mathrm{CS}+\mathrm{LI})$ than when it is unexpected $(\mathrm{CS}-\mathrm{Li})$ is consistent with other demonstrations that the expectation of a drug treatment can reduce its impact. For example, morphine has been shown to produce less analgesia and hyperthermia when it is expected as compared to when it is unexpected (Siegel, 1975, 1976, 1977, 1978; Siegel, Hinson, \& Krank, 1978), and weaker conditioned aversions and less unconditioned suppression of drinking have been observed with expected as compared to unexpected lithium treatment (Domjan, Gillan, \& Gemberling, 1980; Gillan \& Domjan, 1977). Comparable observations have been made with nonpharmacological unconditioned stimuli. Expected nondrug USs have been shown to be less effective than unexpected stimulation in a variety of situations, including conditioning (e.g., Kamin, 1969; Wagner \& Terry, 1975), response elicitation (e.g., Kimble \& Ost, 1961), disruption of stimulus processing or rehearsal (Wagner, Rudy, \& Whitlow, 1973), and response cuing (Terry \& Wagner, 1975).

Expected drug treatments may be less effective than unexpected pharmacological stimulation for a variety of reasons. The signal for the drug $(\mathrm{CS}+)$ may directly evoke a conditional compensatory reaction which counteracts the physiological changes the drug would otherwise produce (Siegel, 1975). In contrast, this compensatory or opponent response may occur as an aftereffect of transient drug-like responses initially elicited by the CS + (Domjan, Gillan, \& Gemberling, 1980). The behavioral effects of expected drug treatments also may be diminished because the $\mathrm{CS}+$ primes the pharmacological stimulus in shortterm memory (STM) and this reduces the amount of rehearsal that administration of the drug produces in STM (Wagner, 1976, 1978). The present findings cannot distinguish among these and other possibilities.

\section{REFERENCE NOTES}

1. Domjan, M., \& Best, M. R. Comparison of the effects of exteroceptive drug-administration cues on the remote and the proximal US preexposure phenomena in taste-aversion learning. Manuscript in preparation, 1979.

2. Best, M. R., \& Domjan, M. Characteristics of the proximal US preexposure effect in taste-aversion learning. Paper presented at the meeting of the Psychonomic Society, San Antonio, November 1978 .

\section{REFERENCES}

Barker, L. M., Best, M. R., \& Domjan, M. (Eds.), Learning mechanisms in food selection. Waco, Tex: Baylor University Press, 1977. 
Best, M. R., \& Domjan, M. Characteristics of the lithiummediated proximal US preexposure effect in flavor-aversion conditioning. Animal Learning \& Behavior, 1979, 7, 433-440.

Cannon, D. S., Berman, R. F., Baker, T. B., \& Atkinson, C. A. Effect of preconditioning unconditioned stimulus experience on learned taste aversions. Journal of Experimental Psychology: Animal Behavior Processes, 1975, 1, 270-284.

Domjan, M. Effects of proximal unconditioned stimulus preexposure on ingestional aversions learned as a result of taste presentation following drug treatment. Animal Learning \& Behavior, $1978,6,133-142$.

Domjan, M., \& Best, M. R. Paradoxical effects of proximal unconditioned stimulus preexposure; Interference with and conditioning of taste aversion. Journal of Experimental Psychology: Animal Behavior Processes, 1977, 3, 310-321.

Domjan, M., Gillan, D. J., \& Gemberling, G. A. Aftereffects of lithium-conditioned stimuli on consummatory behavior in the presence and absence of the drug. Journal of Experimental Psychology: Animal Behavior Processes, 1980, 6, 49-64.

Domjan, M., \& Wilson, N. E. Contribution of ingestive behaviors to taste-aversion learning in the rat. Journal of Comparative and Physiological Psychology, 1972, 80, 403-412.

Gillan, D. J., \& DomJan, M. Taste-aversion conditioning with expected versus unexpected drug treatments. Journal of Experimental Psychology: Animal Behavior Processes, 1977, 3, 297-309.

Kamin, L. J. Predictability, surprise, attention and conditioning. In R. Church \& B. A. Campbell (Eds.), Punishment and aversive behavior. New York: Appleton-Century-Crofts, 1969.

KimBle, G. A., \& OsT, J. W. P. A conditioned inhibitory process in eyelid conditioning. Journal of Experimental Psychology, $1961,61,150-156$.

Mikulka, P. J., Leard, B., \& Klein, S. B. Illness-alone exposure as source of interference with the acquisition and retention of a taste aversion. Journal of Experimental Psychology: Animal Behavior Processes, 1977, 3, 189-201.

Milgram, N. W., Krames, L., \& Alloway, T. M. (Eds.), Food aversion learning. New York: Plenum Press, 1977.

Revusky, S. H., Parker, L., Coombes, J., \& Coombes, S. Rat data which suggest alcoholic beverages should be swallowed during chemical aversion therapy, not just tasted. Behavior Research and Therapy, 1976, 14, 189-194.
SIEGEL, S. Evidence from rats that morphine tolerance is a learned response. Journal of Comparative and Physiological Psychology, 1975, 89, 498-506.

SIEGEL, S. Morphine analgesic tolerance: Its situation specificity supports a Pavlovian conditioning model. Science, 1976, 193. 323.325.

SIEGEL, S. Morphine tolerance acquisition as an associative process. Journal of Experimental Psychology: Animal Behavior Processes, 1977, 3, 1-13.

Siegel, S. Tolerance to the hyperthermic effect of morphine in the rat is a learned response. Journal of Comparative and Physiological Psychology, 1978, 92, 1137-1149.

Siegel, S., Hinson, R. E., \& Krank, M. D. The role of predrug signals in morphine analgesic tolerance: Support for a Pavlovian conditioning model of tolerance. Journal of Experimental Psychology: Animal Behavior Processes, 1978, 4, 188-196.

TERRY, W. S., \& WAGNER, A. R. Short-term memory for "surprising" versus "expected" unconditioned stimuli in Pavlovian conditioning. Journal of Experimental Psychology: Animal Behavior Processes, 1975, 1, 122-133.

Wagner, A. R. Priming in STM: An information processing mechanism for self-generated or retrieval-generated depression of performance. In T. J. Tighe \& R. N. Leaton (Eds.), Habituation: Perspectives from child development, animal behavior, and neurophysiology. Hillsdale, N.J: Erlbaum, 1976.

WAGNER, A. R. Expectancies and the priming of STM. In S. H. Hulse, H. Fowler, \& W. K. Honig (Eds.), Cognitive aspects of animal behavior. Hillsdale, N.J: Erlbaum, 1978.

Wagner, A. R., Rudy, J. W., \& Whitlow, J. W. Rehearsal in animal conditioning. Journal of Experimental Psychology, 1973, 97, 407-426. (Monograph)

Wagner, A. R., \& Terry, W. S. Backward conditioning to a CS following an expected vs. a surprising UCS. Animal Learning \& Behavior, 1975, 3, 370-374.

WILLNER, J.A. Blocking of a taste aversion by prior pairings of exteroceptive stimuli with illness. Learning and Motivation, $1978,9,125-140$

(Received for publication May 31, 1979; revision accepted October $18,1979$. ) 